\title{
Combined Control of Primary and Secondary Suspension of Maglev Vehicles
}

\author{
Ken WATANABE \\ Senior Researcher,
}

\begin{abstract}
Hiroshi YOSHIOKA
Senior Researcher, Laboratory Head,

Erimitsu SUZUKI

Assistant Senior Researcher, Maglev Systems Technology, Research and Development Div., Maglev Systems Development Dept.

To make the superconducting magnetically levitated (maglev) transport system more attractive, it has been important to enhance the ride comfort by controlling vehicle vibration. We have reduced maglev vehicle vibration by controlling only the secondary suspension between the car bodies and bogies. However, by doing so, it has been difficult to reduce vibration for the characteristic and relatively high frequencies of the primary suspension. Recently, we have directed our attention to primary suspension control, using power collection coils of the distributed-type linear generator system, which we are testing as an onboard power source. Because this type of vibration control can apply damping directly to the primary suspension, we consider it to be optimal for reducing vibrations at high frequencies. We have used maglev vehicle models that focus on lateral, vertical, rolling, and pitching motions to describe the effectiveness of reducing vibrations by using linear generator system damping force control in the primary suspension and linear quadratic $(L Q)$ control of the actuators in the secondary suspension.
\end{abstract}

Keywords: Maglev vehicle, vibration control, active suspension, distributed-type linear generator

\section{Introduction}

In the superconducting magnetically levitated (maglev) transport system, passive damping is negligible in the primary suspension between the bogies and ground coils. Because the vehicles consist of lightweight car bodies and bogies equipped with comparatively heavyweight superconducting magnets (SCMs), application of vibration control is expected to be very effective in improving the ride comfort. Moreover, vibration control can reduce the need for extremely precise ground-coil alignment and strict guideway maintenance standards, which in turn would reduce operating costs.

In our previous research, we focused on the secondary suspension between the car bodies and bogies, and examined various types of vibration control methods. We then proposed a system that actively generates electromagnetic damping forces in the primary suspension by running reactive current through power collection coils of a linear generator device that serves as an onboard power supply [1], confirming the characteristics of this system in fundamental experiments [2]. By using this method, the vibrations at the characteristic frequencies of the primary suspension can be reduced, which in principle is difficult to achieve by controlling merely the secondary suspension. Furthermore, the system can be simplified because vibration control can be integrated into the power collection device, which can generate vertical and lateral damping forces by varying the phase of the current flowing through the power collection coils.

In this report, we examine and compare several control method permutations to reduce vibrations in both primary and secondary suspensions, using one maglev vehicle model to focus on lateral and rolling motions, and a second model for vertical and pitching motions.

\section{Control of lateral and rolling motion vibration}

\subsection{Computational model for lateral and rolling motions}

Our simulations were modeled on a maglev vehicle consisting of one car body and one bogie, with a total of four degrees of freedom in the lateral and rolling motions, as shown in Fig. 1 [3]. The secondary suspension is com-

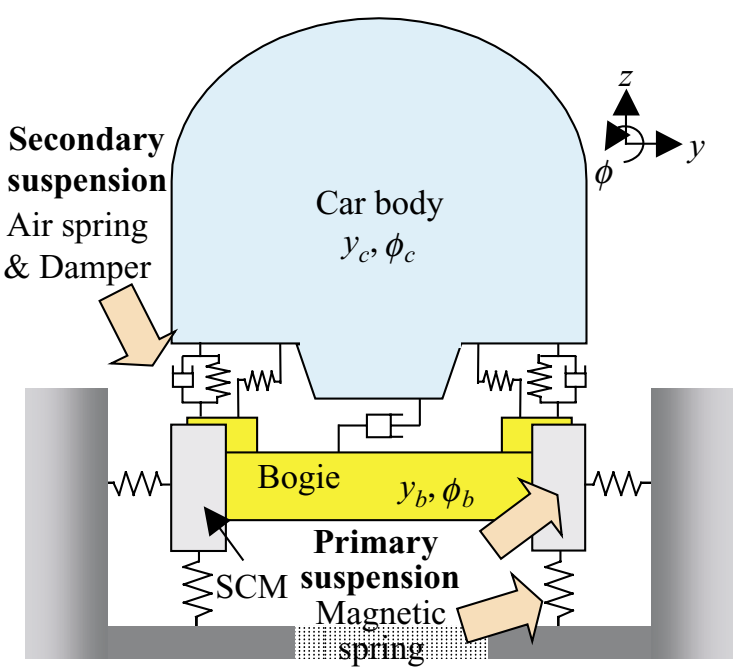

Fig. 1 Computational model for lateral and rolling motions (4-dof) 
prised of air springs and dampers that link the car body to the bogie. The primary suspension relies on the electromagnetic spring interaction between the SCMs attached to the bogie and the levitation coils attached to the sidewalls of the guideway. External disturbances were applied to the vehicle by irregularities in the lateral alignment of these levitation coils, the lateral and rolling motions of the primary electromagnetic suspension being coupled with each other.

We examined the following three cases:

Case L1: Linear quadratic (LQ) control of lateral motions in the secondary suspension

Case L2: LQ control of lateral and rolling motions in the secondary suspension

Case L3: LQ control of lateral and rolling motions in the secondary suspension (Case L2), combined with electromagnetic damping control of lateral motions in the primary suspension

In our model, parameter values for which are shown in Table 1, we used actuators in place of the dampers to control the secondary suspension, and electromagnetic damping forces generated by the distributed-type linear generator to control the primary suspension, assuming that these would be ideal with no time delay. Two vertical actuators generated the rolling motion of the car body. Model specifications were derived from a reference [4].

Table 1 Model parameters

\begin{tabular}{ll}
\hline \multicolumn{1}{c}{ Parameter } & \multicolumn{1}{c}{ Value } \\
\hline Bogie mass & $6.0 \times 10^{3}[\mathrm{~kg}]$ \\
Car body mass & $14.0 \times 10^{3}[\mathrm{~kg}]$ \\
Air spring lateral spring constant & $6.0 \times 10^{5}[\mathrm{~N} / \mathrm{m}]$ \\
Air spring vertical spring constant & $2.0 \times 10^{5}[\mathrm{~N} / \mathrm{m}]$ \\
Lateral damper damping constant & $5.0 \times 10^{4}[\mathrm{Ns} / \mathrm{m}]$ \\
Vertical damper damping constant & $5.0 \times 10^{3}[\mathrm{Ns} / \mathrm{m}]$ \\
Magnetic spring lateral spring constant & $3.0 \times 10^{6}[\mathrm{~N} / \mathrm{m}]$ \\
Magnetic spring rolling spring constant & $1.0 \times 10^{7}[\mathrm{~N} / \mathrm{rad}]$ \\
Magnetic spring vertical spring constant & $4.7 \times 10^{6}[\mathrm{~N} / \mathrm{m}]$ \\
Magnetic spring pitching spring constant & $1.0 \times 10^{7}[\mathrm{~N} / \mathrm{rad}]$ \\
\hline
\end{tabular}

\subsection{Secondary suspension control design - lateral and rolling motions}

The objective of the secondary suspension control is to reduce the lateral and rolling accelerations of the car body and to maintain the gap between the bogies and the guideway. We adopted the LQ control theory for the secondary suspension.

The state variables of the model were arranged in the vector $x$ as:

$$
x=\left[\begin{array}{llllllll}
y_{c} & \dot{y}_{c} & y_{b} & \dot{y}_{b} & \phi_{c} & \dot{\phi}_{c} & \phi_{b} & \dot{\phi}_{b}
\end{array}\right]^{T}
$$

Here, $y$ and $\phi$ represent lateral and rolling angular displacements, respectively. The subscript $b$ stands for the bogie, and $c$ stands for the car body.

The state equation is:

$$
\dot{x}=A x+B u+W y_{0}
$$

Here, $A, B$, and $W$ represent the system state, control, and disturbance matrices, respectively. The variable $u$ is the control input, and $y_{0}$ is the lateral irregularities in the alignment of the coils on the guideway sidewalls.

The evaluation function $J$ is expressed as:

$$
J=\int_{0}^{\infty}\left[\left(\frac{\ddot{y}_{c}}{q_{1}}\right)^{2}+\left(\frac{y_{b}}{q_{2}}\right)^{2}+\left(\frac{\ddot{\phi}_{c}}{q_{3}}\right)^{2}+\left(\frac{\phi_{b}}{q_{4}}\right)^{2}+\left(\frac{u}{r}\right)^{2}\right] d t
$$

where $q_{1}$ through $q_{4}$, and $r$ are weighting factors.

We found the values of these weighting factors that minimize this evaluation function, and derived the feedback gain $K$.

Finally, the control input $u$ is expressed as:

$$
u=-K x
$$

\subsection{Primary suspension control design - lateral motions}

Because the maximum damping force that can be generated is relatively small, we chose a simple ON/OFF control (Eq. 5) for the primary suspension to fully utilize this small damping force, generating the Coulomb damping effect. The direction of the damping force adapts to the sign of the lateral velocity of the bogie. The lateral damping force $F_{m y}$ is expressed as:

$$
F_{m y}=\operatorname{sign}\left(\dot{y}_{b}\right) \cdot f_{m y}
$$

where $f_{m y}$ represents the maximum possible lateral damping force generated by the power collection system.

\subsection{Case L1: LQ control of lateral motion in the secondary suspension}

Figure 2 shows examples of frequency responses of the car body vibrations in the case of LQ control of lateral motion in the secondary suspension. Lateral actuators were used to control the motions. Responses for a passive model are also shown for comparison. The lefthand graph of Fig. 2 confirms that the gain of lateral acceleration is markedly reduced in the frequency range of 0.8 to $2 \mathrm{~Hz}$. The peak in the 2 to $3 \mathrm{~Hz}$ frequency range, which represents the characteristic frequency of the coupled motion of the car body and bogie, increased slightly in both the lateral and rolling angular accelerations.

\subsection{Case L2: LQ control of lateral and rolling motions in the secondary suspension}

Figure 2 also provides examples of frequency responses of the car body vibrations in the case of LQ control of lateral and rolling motions in the secondary suspension. Lateral and rolling actuators were used to control the motions. The left-hand graph of Fig. 2 confirms that the gain of lateral acceleration is again markedly reduced in the frequency range of 0.8 to $2 \mathrm{~Hz}$, as in Case L1. Furthermore, the right-hand graph of Fig. 2 shows that the rolling angular accelerations are also reduced in the same frequency range. In other words, adding rolling motion control improved the rolling angular accelerations with no adverse effect on the lateral accelerations. 

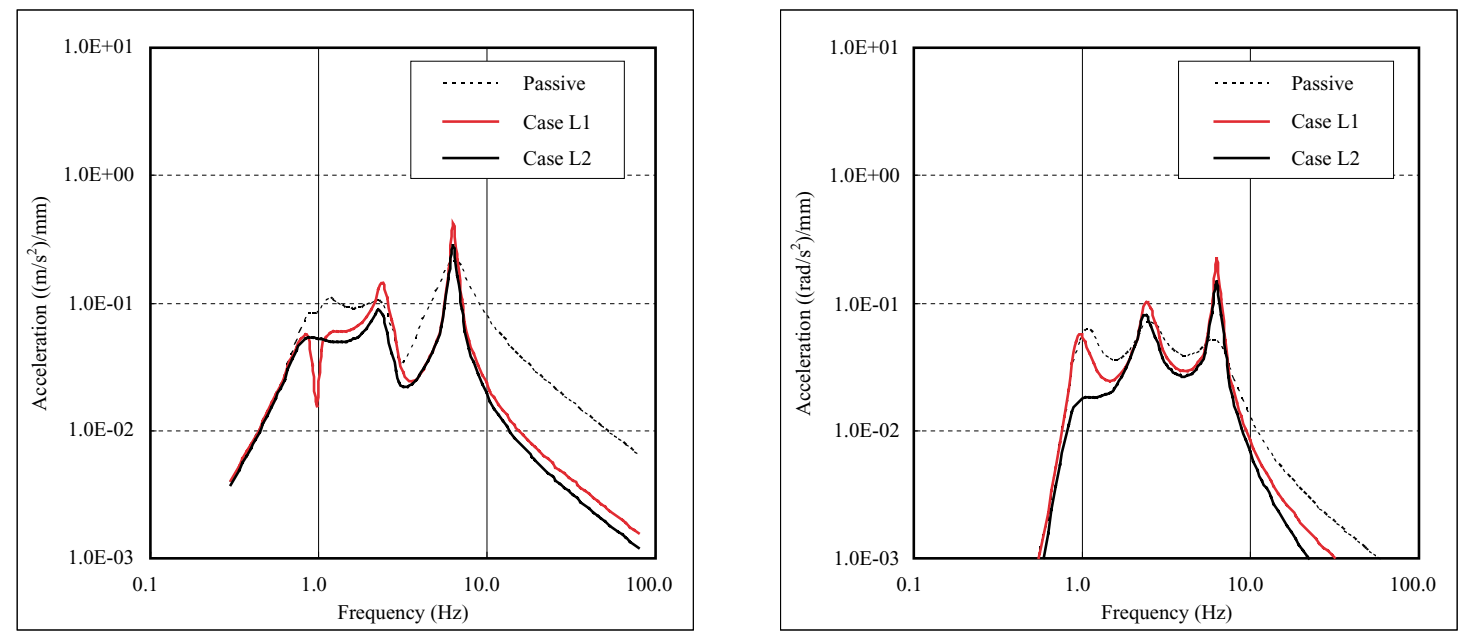

Fig. 2 Frequency responses for Cases L1 and L2 in comparison with the passive case (left: lateral acceleration of car body, right: rolling angular acceleration of car body)

2.6 Case L3: LQ control of lateral and rolling motions in the secondary suspension, combined with electromagnetic damping control of lateral motions in the primary suspension

Figure 3 shows examples of frequency responses of the car body vibrations for the case of LQ control of lateral and rolling motions in the secondary suspension, combined with electromagnetic damping control of lateral motions in the primary suspension. For both the translational accelerations of the lateral motion and the angular accelerations of the rolling motion, this control reduces the peaks of acceleration near the characteristic vibration frequencies of both primary and secondary suspensions. This combined control reduces vibrations in the widest range of frequencies and can therefore be expected to improve ride comfort.

\section{Control of vertical and pitching motion vibration}

\subsection{Computational model for vertical and pitching} motions

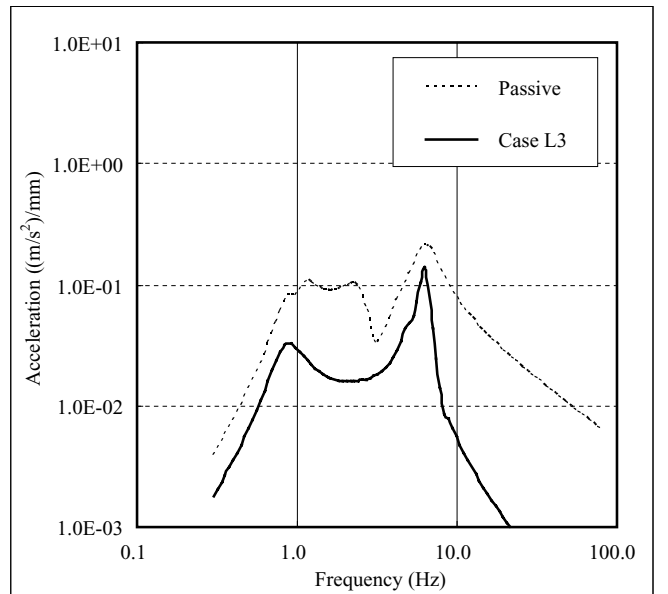

Our simulations modeled a maglev train set consisting of three car bodies and two articulated bogies with a total of ten degrees of freedom in the vertical and pitching motions, as shown in Fig. 4 [5]. Each car body was considered to be rigid, the front of car body No. 1 and the rear of car body No. 3 being supported by air springs and dampers attached to stationary walls. Car body No. 2 was attached to bogies at both the front and rear. The car bodies were connected to the bogies with air springs and dampers.

Once again, we used actuators in place of the dampers to control the secondary suspension, and electromagnetic damping forces generated by the distributed-type linear generator to control the primary suspension. We then simulated the vibration control of car body No. 2 .

We examined the following three cases:

Case V1: LQ control of vertical and pitching motions in the secondary suspension

Case V2: Electromagnetic damping control of vertical motions in the primary suspension

Case V3: LQ control of vertical and pitching motions in the secondary suspension (Case V1), combined with electromagnetic damping control of ver-

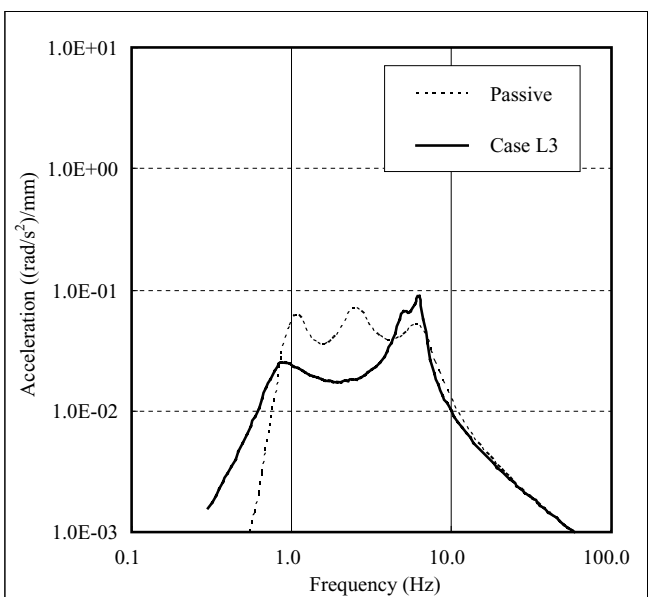

Fig. 3 Frequency response for Case L3 in comparison with the passive case (left: lateral acceleration of car body, right: rolling angular acceleration of car body) 


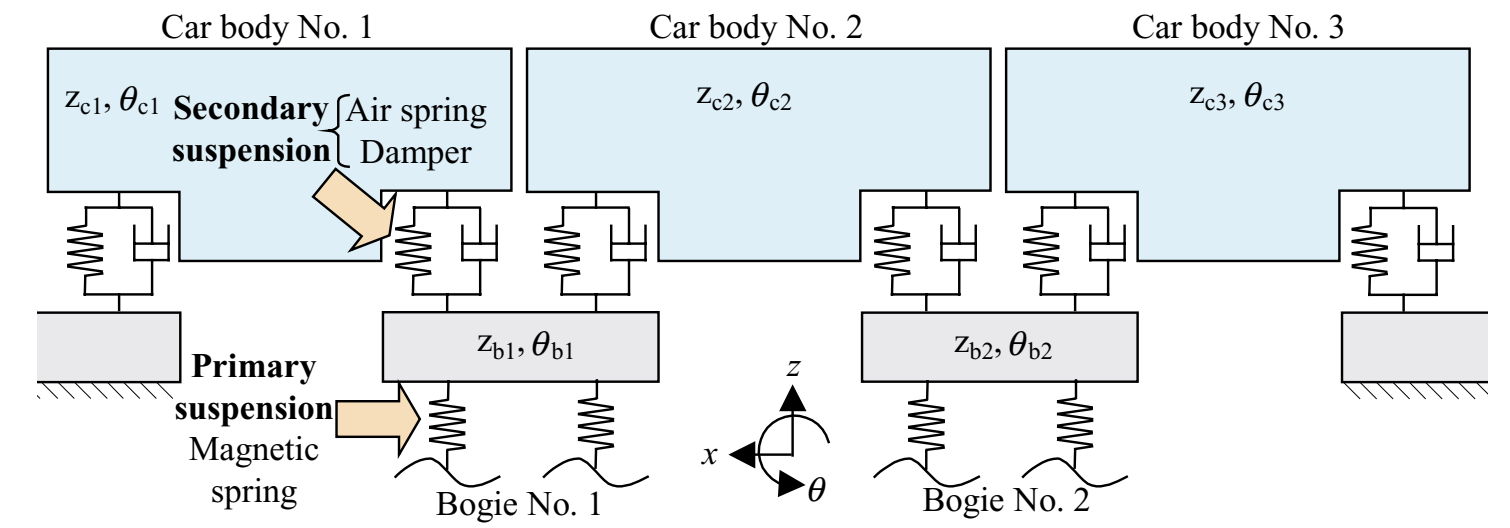

Fig. 4 Computational model for vertical and pitching motions (10-dof)

tical motions in the primary suspension (Case V2)

The actuators were again assumed to be ideal, with no delay in response. Vertical irregularities in the alignment of the ground coils were added to serve as the source of external disturbances, the magnitudes of misalignment being input at two points, the front and rear of each bogie. Aerodynamic influences were not taken into account.

In the primary suspension, magnetic spring characteristics in the vertical and pitching directions for a vehicle running at $500 \mathrm{~km} / \mathrm{h}$ were represented by two vertical springs, at the front and rear of the bogies. The coupled vertical and pitching component of the magnetic spring was small and therefore assumed to be negligible. As in the control of the lateral and rolling motions, we used the specifications of the model from a reference [4] and used the values for the parameters of the model shown in Table 1.

\subsection{Secondary suspension control design - vertical and pitching motions}

Taking into account the time delay between inputs of external disturbances on the bogies in the control of the secondary suspension, the control device for bogies No. 1 and No. 2 was designed as one unit. Specifically, we used the input of external disturbances at the front of bogie No. 1 as a reference, and estimated the time delays for the inputs of disturbance on the remaining three inputs (at the rear of bogie No.1 and the front and rear of bogie No. 2) using seventh-order Padé approximations.

Figure 5 shows examples of frequency responses of the vertical vibrations at the center of weight of car body No. 2 for a passive model. The frequency responses to inputs of external disturbances using conventional complex amplitudes are compared with those of disturbances incorporating the approximated time delays mentioned previously. Peaks in the 1 to $2 \mathrm{~Hz}$ range correspond to characteristic vibration frequencies of the secondary suspension, those in the 4 to $5 \mathrm{~Hz}$ range corresponding to characteristic vibration frequencies of the primary suspension. The acceleration becomes low in the region around $3 \mathrm{~Hz}$ because the input there corresponds to a wavelength that is approximately twice the length of the car body. It can be seen that the approximation is in agreement with the conventional method for frequencies up to $10 \mathrm{~Hz}$ and thus poses no problems in calculating vibrations for frequencies below $10 \mathrm{~Hz}$. However, higher orders of approximations may be necessary when considering bending and other motions of the car body at frequencies above $10 \mathrm{~Hz}$.

In the control of the secondary suspension, we installed idealized vertical actuators between the car body and bogie, and applied LQ control. The evaluation function incorporated the vertical accelerations of the center of the car body, the vertical accelerations of the ends of the car body caused by pitching motion, and relative displacements between the car body and bogie at the positions of the air springs (Eq. 6).

$$
J=\int_{0}^{\infty}\left[\left(\frac{\ddot{z}_{c 2}}{q_{1}}\right)^{2}+\left(\frac{\ddot{\theta}_{c 2}}{q_{2}}\right)^{2}+\left(\frac{\Delta z_{1}}{q_{3}}\right)^{2}+\left(\frac{\Delta z_{2}}{q_{4}}\right)^{2}+\left(\frac{u}{r}\right)^{2}\right] d t
$$

Here, $z_{c 2}$ and $\theta_{c 2}$ represent the vertical and pitching angular displacements of car body No. 2, respectively, and $u$ is the control input. The variables $\Delta z_{1}$ and $\Delta z_{2}$ are the displacements of car body No. 2 relative to bogies No. 1 and No. 2. Again as in the lateral and rolling motions, $q_{1}$ through $q_{4}$, and $r$ are weighting factors.

We found the solution for the LQ equation that mini-

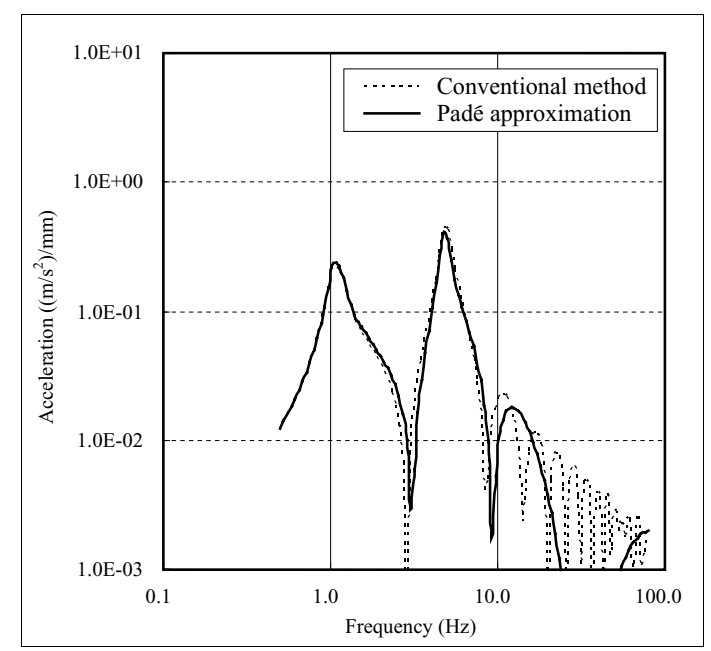

Fig. 5 Comparison of frequency responses using the conventional method and Padé approximation (vertical acceleration of car body center) 

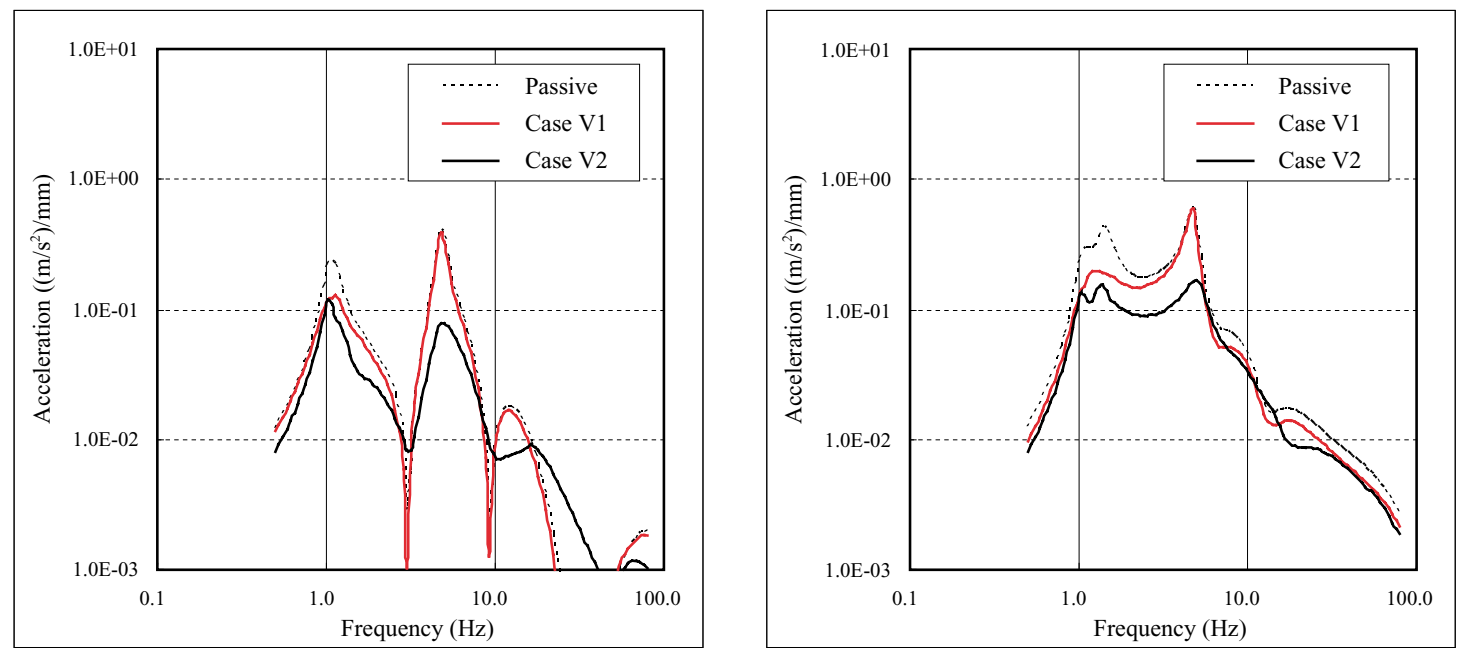

Fig. 6 Frequency responses for Cases V1 and V2 in comparison with the passive case (left: vertical acceleration of car body center, right: vertical acceleration of end of car body)

mizes this evaluation function, derived the feedback gain and set the weighting factors of the evaluation function to reduce the peaks of the accelerations near the characteristic vibration frequencies in the primary suspension. At the same time, we confirmed the values to be sufficiently conservative, so that the displacement between car body and bogie does not become large enough to cause the vertical motion stoppers to hit the limits. Satisfying these conditions, we decided on the weighting factors to minimize the required magnitude of the controlling force.

\subsection{Primary suspension control design - vertical motions}

It was assumed that we were able to make use of the vertical damping force generated by the power collection system mentioned previously to control the primary suspension. An ON/OFF control (Eq. 7) similar to that used for lateral motion control was used, the direction of the generated damping force adapting to the sign of the vertical velocity of the bogie [3].

$$
F_{m z}=\operatorname{sign}\left(\dot{z}_{b}\right) \cdot f_{m z}
$$

Here, $F_{m z}$ represents the controlling force, and $f_{m z}$ the maximum possible vertical damping force generated by the power collection system.

We assumed that the controlling forces of bogies No. 1 and No. 2 could be added independently. Because the $\mathrm{ON} / \mathrm{OFF}$ control is non-linear and the frequency response could not be directly calculated, we used a time-dependent simulation in the computations.

\subsection{Case V1: LQ control of vertical and pitching motions in the secondary suspension}

Figure 6 shows examples of frequency responses of the car body vibrations for the case of $L Q$ control of vertical and pitching motions in the secondary suspension. The graphs indicate that the control of the secondary suspension has reduced the peaks near the characteristic vibration frequencies of the secondary suspension in the 1 to $2 \mathrm{~Hz}$ range, confirming the effectiveness of the method in controlling vibrations. The right-hand graph shows that the control was also effective in reducing such peaks at the end points of the car body. Therefore, a combined control using the actuators of bogies No. 1 and No. 2 can control vibrations of the pitching motion.

On the other hand, the control had almost no effect on the peaks near the characteristic vibration frequencies of the primary suspension in the 4 to $5 \mathrm{~Hz}$ range, indicating the limitations of the control of the secondary suspension alone. The characteristic vibration frequencies of the primary suspension are within the 4 to 8 $\mathrm{Hz}$ range, which is the most sensitive region according to the ISO-2631 frequency weighting curves used to evaluate ride comfort. Therefore, we consider reducing vibrations of the primary suspension to be effective in improving the ride comfort for vertical motions.

\subsection{Case V2: Electromagnetic damping control of vertical motions in the primary suspension}

Figure 6 also shows examples of frequency responses of the car body vibrations with electromagnetic damping control of vertical motions in the primary suspension. In the case of the car body center shown in the lefthand graph of Fig. 6, we applied vertical damping forces of $3 \mathrm{kN}$ to the bogie. This control greatly reduced the peaks near the characteristic frequencies of the magnetic springs in the 4 to $5 \mathrm{~Hz}$ range, suggesting that the control of the primary suspension alone can effectively reduce vibrations even with a small generated damping force.

Furthermore, in the frequency response computations, we input vertical irregularities in the alignment of the ground coils by using wave patterns with magnitudes of $\pm 1 \mathrm{~mm}$. In this model, the vertical motions of the bogie decrease and the pitching motions become dominant when the input vertical damping forces are $4 \mathrm{kN}$ or higher. 

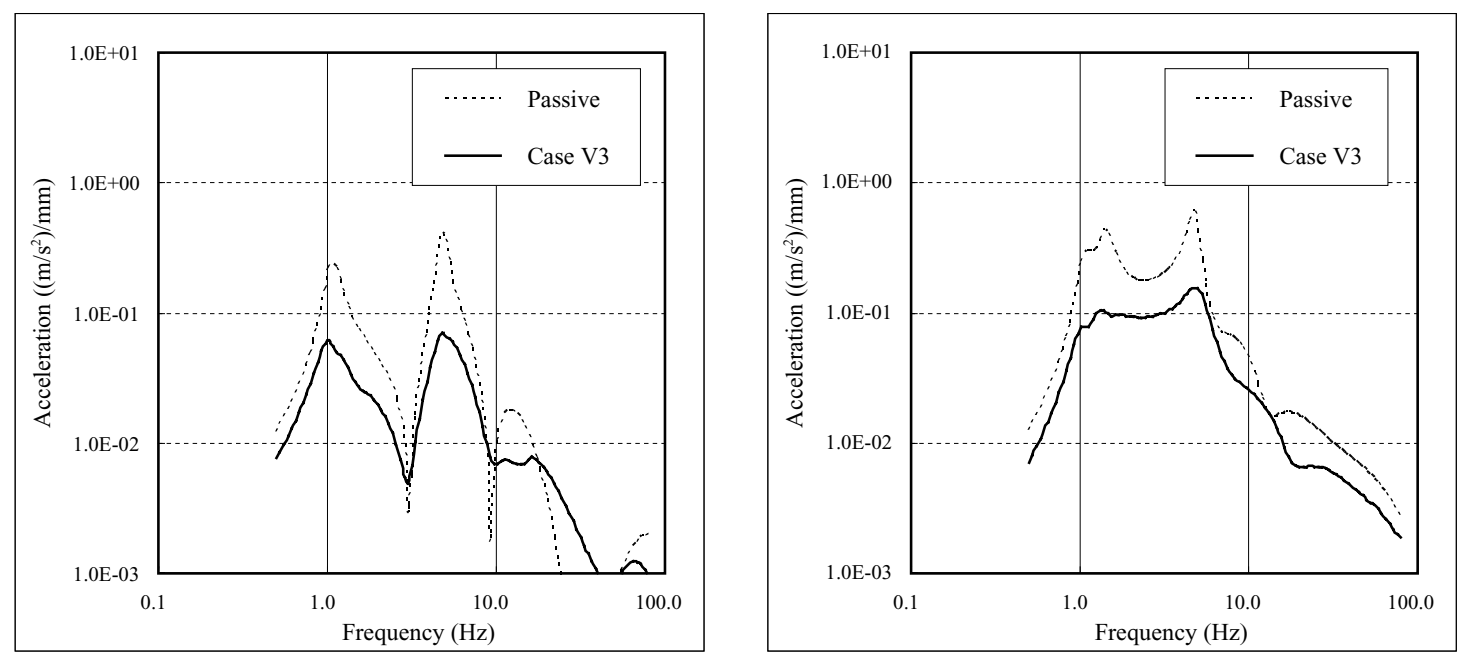

Fig. 7 Frequency response for Case V3 in comparison with the passive case (left: vertical acceleration of car body center, right: vertical acceleration of end of car body)

3.6 Case V3: LQ control of vertical and pitching motions in the secondary suspension, combined with electromagnetic damping control of vertical motions in the primary suspension

Figure 7 shows examples of frequency responses of the car body vibrations with LQ control of vertical and pitching motions in the secondary suspension, combined with electromagnetic damping control of vertical motions in the primary suspension. This combined control reduces the peaks of acceleration near the characteristic vibration frequencies of both the primary and secondary suspensions at both the center and end points of the car body, and is expected to considerably improve ride comfort.

\section{Conclusions}

We have demonstrated the validity and effectiveness of combing control methods to improve the vibration control of the primary and secondary suspension, using models to simulate lateral and rolling motions, as well as vertical and pitching motions of the superconducting maglev vehicles. The combined control system reduced vibrations even in higher frequency ranges, suggesting that it can considerably improve ride comfort. Such improvements to the vehicle will be useful in reducing maintenance and construction costs of the ground coils and guideway and enhance the commercial operation of the superconducting maglev system. Possibilities for the adaptation of other control laws and further maglev model details are currently being studied.

\section{References}

1) Murai, T., Hasegawa, H., Yamamoto, T. and Fujiwara, S.: Active Magnetic Damper Using Linear Generator, Trans. IEE Japan, Vol. 119-D, No. 11, 1371-1376 (1999, Japanese).

2) Hasegawa, H., Murai, T., Yamamoto, T. and Matsue, H.: Development of Combined SC Type Linear Generator, Proc. $14^{\text {th }}$ Symposium on Electromagnetics and Dynamics, 263-264 (2002, Japanese).

3) Watanabe, K., Yoshioka, H., Watanabe, E., Nagai, M. and Tohtake, T.: A Study of the Vibration Control System for a Superconducting Maglev Vehicle, Proc. $6^{\text {th }}$ Int'l Conference on Motion and Vibration Control (MOVIC), Vol. 2, 907-912 (2002, English).

4) Azakami, M.: The Development of Maglev Bogie System on the First Train Set for Yamanashi Test Line, RTRI Report, Vol. 10, No. 1, 11-16 (1996, Japanese).

5) Watanabe, K., Yoshioka, H., Suzuki, E., Watanabe, E., Nagai, M. and Tohtake, T.: A Study of Combined Control of Primary and Secondary Suspension of a Maglev Vehicle, Proc. Int'l Symposium on Speed-up and Service Technology for Railway and Maglev Systems 2003 (STECH'03), 467-471 (2003, English). 